\title{
Spin Calogero models obtained from dynamical $r$-matrices and geodesic motion
}

\author{
L. FEHÉR ${ }^{a}$ and B.G. PUSZTAI ${ }^{b}$ \\ ${ }^{a}$ Department of Theoretical Physics, MTA KFKI RMKI \\ 1525 Budapest 114, P.O.B. 49, Hungary, and \\ Department of Theoretical Physics, University of Szeged \\ Tisza Lajos krt 84-86, H-6720 Szeged, Hungary \\ e-mail: lfeher@rmki.kfki.hu \\ ${ }^{b}$ Centre de recherches mathématiques, Université de Montréal \\ C.P. 6128, succ. centre ville, Montréal, Québec, Canada H3C 3J7, and \\ Department of Mathematics and Statistics, Concordia University \\ 7141 Sherbrooke W., Montréal, Québec, Canada H4B 1R6 \\ e-mail: pusztai@CRM.UMontreal.CA
}

\begin{abstract}
We study classical integrable systems based on the Alekseev-Meinrenken dynamical $r$-matrices corresponding to automorphisms of self-dual Lie algebras, $\mathcal{G}$. We prove that these $r$-matrices are uniquely characterized by a non-degeneracy property and apply a construction due to $\mathrm{Li}$ and $\mathrm{Xu}$ to associate spin Calogero type models with them. The equation of motion of any model of this type is found to be a projection of the natural geodesic equation on a Lie group $G$ with Lie algebra $\mathcal{G}$, and its phase space is interpreted as a Hamiltonian reduction of an open submanifold of the cotangent bundle $T^{*} G$, using the symmetry arising from the adjoint action of $G$ twisted by the underlying automorphism. This shows the integrability of the resulting systems and gives an algorithm to solve them. As illustrative examples we present new models built on the involutive diagram automorphisms of the real split and compact simple Lie algebras, and also explain that many further examples fit in the dynamical $r$-matrix framework.
\end{abstract}




\section{Introduction}

The study of integrable many body systems initiated by Calogero [1, Sutherland [2] and Moser [3] is popular since these systems have interesting applications in several branches of theoretical physics and involve fascinating mathematics. See, for example, the reviews in 4, 5, 6, 7,. The generalized Calogero models associated to root systems by Olshanetsky and Perelomov [8], the spin Calogero models due to Gibbons and Hermsen [9], and the Ruijsenaars-Schneider models [10] are also very important. Many approaches were applied to prove the classical integrability of these models. The most general among these are the construction of a Lax representation for the equation of motion and the realization of the system as a 'projection' of some other system that is integrable 'obviously', for example the free geodesic motion on a Lie group or on a symmetric space (see [3, 11, 12, and the review [4]). The result of Babelon and Viallet [13] linking Liouville integrability to the form of the Poisson brackets (PBs) of the Lax matrix is also relevant for us, since the determination of the $r$-matrices encoding these PBs in Calogero models led eventually to the questions investigated in this paper.

The $r$-matrices of the $A_{n}$ type spinless Calogero models found in [14, 15, 16] depend on the coordinates of the particles and are not unitary. They were re-derived by Avan, Babelon and Billey in [17] using Hamiltonian reduction of an auxiliary non-integrable spin extension of the Calogero model. The definition of the non-integrable model relies on a 'quasi-Lax operator', whose PBs (of the type (2.10) below) are encoded by certain unitary dynamical $r$-matrices that yield the Calogero $r$-matrices upon reduction. The unitary $r$-matrices in question were later recognized [18 to be identical to the fundamental solutions of the so-called classical dynamical Yang-Baxter equation (CDYBE) that appeared first in studies of the WZNW conformal field theory [19, 20]. Subsequently, the geometric meaning of the CDYBE and the classification of its solutions was investigated by Etingof and Varchenko [21], and dynamical $r$-matrices have since turned up in several contexts.

The 'wonderful but mysterious' calculations of [17] were understood on an abstract level by $\mathrm{Li}$ and $\mathrm{Xu}$, who then proposed a method to associate an integrable spin Calogero type model to any spectral parameter dependent [22] or independent [23] dynamical $r$-matrix defined in [21]. In our view the essential point in [22, 23] is that a 'quasi-Lax operator' of a non-integrable spin Calogero type system can be directly associated with any solution of the CDYBE, and upon Hamiltonian reduction this yields a model admitting a Lax representation in the usual sense. $\mathrm{Li}$ and $\mathrm{Xu}$ also constructed new spin Calogero models by applying their method to the rational, trigonometric and elliptic solutions of the CDYBE given in [21. (The spin variables can be rendered frozen only in the $A_{n}$ case.) A companion construction of spin Ruijsenaars models was presented in [24]. These results (see also [25, 26]) are significant and deserve further study.

The main purpose of the present paper is to characterize the integrable spin Calogero type models that may be associated with the remarkable classical dynamical $r$-matrices found by Alekseev and Meinrenken in [27. These trigonometric solutions of the CDYBE are in correspondence with scalar product preserving automorphisms of self-dual Lie algebras. In particular, they contain $r$-matrices labeled by the automorphisms of the Dynkin diagrams of the simple Lie algebras, and it seems interesting to enquire about the corresponding integrable 
systems. In our investigation we shall proceed in such a way to underline that the association of the spin Calogero type models to the solutions of the CDYBE does not actually rely on the machinery of Lie algebroids that features in [22, 23], but rather it can be done in a very direct manner. This does not mean that the algebroid-groupoid technique is not important, since it plays a crucial role in the factorization algorithm worked out in [23, 24, 26] to solve the resulting models in the general (including the elliptic) case. Still, as these techniques are not widely known, it is worth stressing that in some respects they are not essential.

In our case the algebroid-groupoid technique can also be bypassed since it will be demonstrated that the models associated with the Alekseev-Meinrenken $r$-matrices (3.2) can be viewed as projections of the natural geodesic system on a corresponding Lie group. We first show this at the level of the equation of motion, which immediately yields a solution algorithm. Then we prove that the spin Calogero phase space obtained from the dynamical $r$-matrix construction is identical to a reduced phase space coming from the cotangent bundle of the corresponding Lie group, which is reduced using the adjoint action twisted by the underlying automorphism. This generalizes Reshetikhin's derivation [28] of the 'principal trigonometric' spin Calogero model by Hamiltonian reduction, which corresponds to the identity automorphism. Certain rational spin Calogero models will be derived in a similar way, too, generalizing results in [5, 29, 30, 31].

As illustrative examples, we shall present new spin Calogero models built on the non-trivial involutive diagram automorphisms of the simple Lie algebras. We shall also point out that many other models can be obtained using scalar product preserving automorphisms of reductive Lie algebras, including for instance certain models found earlier by Blom and Langmann [32] and by Polychronakos [33] by means of different methods. The degenerate complete integrability of the spin Calogero models corresponding to the identity automorphism was explained in [28] from the reduction viewpoint (without making explicit the relationship to dynamical $r$-matrices). It should be possible to generalize the arguments of [28 to our examples, and one may also try to quantize our systems by quantum Hamiltonian reduction.

In the next section, the construction of generalized spin Calogero models based on solutions of the CDYBE is reviewed. Subsequently, this construction is applied to those $r$-matrices that are non-degenerate in a technical sense, defined in Section 3, which ensures that their equation of motion is equivalent to the Lax equation (2.17). This holds for the rational $r$-matrices (3.1) and for the Alekseev-Meinrenken $r$-matrices (3.2). In Section 3 the Lax equation (2.17) is shown to be a projection of the geodesic equation on the underlying Lie algebra or its Lie group in correspondence with the rational and trigonometric cases. Section 4 clarifies the symplectic aspect of this projection. In Section 5 the spin Calogero Hamiltonians associated with the nontrivial involutive diagram automorphisms of the real split and compact simple Lie algebras are presented. After a brief summary, further examples and other issues are discussed in Section 6. In Appendix A the $r$-matrices (3.2) are proved to be uniquely characterized by their nondegeneracy property, which also leads to a simple description of all quasi-triangular solutions of the CDYBE in the standard compact case. Appendix B contains some auxiliary material.

Our main results are Proposition 2 in Section 3 and Proposition 3 in Section 4 concerning the projection method, and the uniqueness statement of Proposition A.2 about the AlekseevMeinrenken $r$-matrices together with its corollary dealing with the standard compact case. 


\section{Spin Calogero models from dynamical $r$-matrices}

We below recapitulate the construction of spin Calogero type models developed by Li and $\mathrm{Xu}$ [22, 23] generalizing the earlier work of Avan, Babelon and Billey [17, 18]. The essential point is contained in Proposition 1 below, which is actually very simple. For our present purpose, we consider only spectral parameter independent dynamical $r$-matrices defined on a self-dual Abelian subalgebra, $\mathcal{K}$, of a self-dual (real or complex) Lie algebra $\mathcal{G}$.

The self-duality of $\mathcal{G}$ means that it is equipped with a non-degenerate, invariant, symmetric bilinear form $\langle$,$\rangle , which is real or complex valued depending on \mathcal{G}$ being real or complex. A subalgebra $\mathcal{K} \subset \mathcal{G}$ is called self-dual if the restriction of $\langle$,$\rangle to \mathcal{K}$ remains non-degenerate. (In the physically most interesting cases $\mathcal{G}$ is a real Lie algebra and $\langle$,$\rangle has definite signature on$ $\mathcal{K}$.) The 'scalar product' $\langle$,$\rangle gives rise to the orthogonal decomposition$

$$
\mathcal{G}=\mathcal{K}+\mathcal{K}^{\perp}, \quad X=X_{\mathcal{K}}+X_{\perp} \quad \forall X \in \mathcal{G}
$$

A dynamical $r$-matrix associated with $\mathcal{K} \subset \mathcal{G}$ is by definition a (smooth or holomorphic) map

$$
R: \check{\mathcal{K}} \rightarrow \operatorname{End}(\mathcal{G})
$$

on some open domain $\check{\mathcal{K}} \subset \mathcal{K}$, which is required to be antisymmetric with respect to $\langle$,$\rangle ,$ equivariant and subject to the CDYBE [21]. Using the adjoint representation of $\mathcal{G}$, for an Abelian $\mathcal{K}$ the (infinitesimal) equivariance property reads

$$
\operatorname{ad}_{\kappa} \circ R(q)=R(q) \circ \operatorname{ad}_{\kappa} \quad \forall q \in \check{\mathcal{K}}, \quad \kappa \in \mathcal{K} .
$$

We introduce dual bases $\left\{T^{i}\right\}$ and $\left\{T_{j}\right\}$ in $\mathcal{K}$, and let $q^{i}=\left\langle q, T^{i}\right\rangle$ denote the corresponding coordinates on $\check{\mathcal{K}}$. Below we use the notation

$$
\nabla_{\kappa} R=\kappa^{i} \frac{\partial R}{\partial q^{i}}, \quad\langle X,(\nabla R) Y\rangle=T^{i}\left\langle X, \frac{\partial R}{\partial q^{i}} Y\right\rangle \quad \forall X, Y \in \mathcal{G} .
$$

The CDYBE ('with coupling $\nu$ ') can be formulated as the condition

$$
E_{\nu}(R, X, Y)=0 \quad \forall X, Y \in \mathcal{G},
$$

where $\nu$ is a constant and

$$
\begin{aligned}
E_{\nu}(R, X, Y): & =\frac{\nu^{2}}{4}[X, Y]+[R X, R Y]-R([X, R Y]+[R X, Y]) \\
& +\langle X,(\nabla R) Y\rangle+\left(\nabla_{Y_{\mathcal{K}}} R\right) X-\left(\nabla_{X_{\mathcal{K}}} R\right) Y .
\end{aligned}
$$

In the 'triangular' case $\nu=0$ and in the 'quasi-triangular' case $\nu=1$.

Let us identify $\mathcal{K}$ and $\mathcal{G}$ with their dual spaces by means of the scalar product, extend the bases $\left\{T^{i}\right\}$ and $\left\{T_{j}\right\}$ of $\mathcal{K}(i, j=1, \ldots, \operatorname{dim}(\mathcal{K}))$ to dual bases $\left\{T^{a}\right\}$ and $\left\{T_{b}\right\}$ of $\mathcal{G}(a, b=$ $1, \ldots, \operatorname{dim}(\mathcal{G}))$, and define $f_{a b}^{c}:=\left\langle\left[T_{a}, T_{b}\right], T^{c}\right\rangle$. Then consider the phase space

$$
\mathcal{M}:=T^{*} \check{\mathcal{K}} \times \mathcal{G}^{*} \simeq \check{\mathcal{K}} \times \mathcal{K} \times \mathcal{G} \simeq\{(q, p, \xi)\}
$$


equipped with the direct product of the natural Poisson brackets $(\mathrm{PBs})$ on $T^{*} \check{\mathcal{K}}$ and on $\mathcal{G}^{*}$. In coordinates,

$$
\left\{q^{i}, p_{j}\right\}=\delta_{j}^{i}, \quad\left\{\xi_{a}, \xi_{b}\right\}=f_{a b}^{c} \xi_{c} .
$$

Let us now introduce the function $L: \mathcal{M} \rightarrow \mathcal{G}$ by

$$
L:(q, p, \xi) \mapsto p-\left(R(q)+\frac{\nu}{2}\right) \xi
$$

One may call $L$ a quasi-Lax operator, because it satisfies PBs of the 'St Petersburg type' up to an 'anomalous term'. This is the content of the following proposition.

Proposition 1 [22, 23]. For any dynamical $r$-matrix $R$ on an Abelian, self-dual subalgebra $\mathcal{K} \subset \mathcal{G}$, the $\mathcal{G}$-valued function $L$ (2.9) on $\mathcal{M}$ verifies the Poisson bracket relation

$$
\left\{L_{1}, L_{2}\right\}=\left[R_{12}, L_{1}+L_{2}\right]-\nabla_{\xi_{\mathcal{K}}} R_{12},
$$

where $\xi_{\mathcal{K}}:(q, p, \xi) \mapsto \xi_{\mathcal{K}}$ is the evaluation map and $R_{12}=\left\langle T_{a}, R T_{b}\right\rangle T^{a} \otimes T^{b}, L_{1}=L \otimes 1$, $L_{2}=1 \otimes L$.

Incidentally, the proof shows a stronger result. Namely, by assuming the antisymmetry and the equivariance properties one can calculate that

$$
\left\{L_{1}, L_{2}\right\}-\left(\left[R_{12}, L_{1}+L_{2}\right]-\nabla_{\xi \mathcal{K}} R_{12}\right)=\left\langle\xi, E_{\nu}\left(R, T^{a}, T^{b}\right)\right\rangle T_{a} \otimes T_{b},
$$

which shows that the CDYBE (2.5) is equivalent to the PB (2.10).

The message from (2.10) is that if the derivative term was absent, then the $\mathcal{G}$-invariant functions of $L$ would provide a Poisson commuting family (as usual in classical integrable systems). Thus one should impose the first class constraints

$$
\xi_{\mathcal{K}}=0
$$

and then the $\mathcal{G}$-invariant functions of $L$ yield a commuting family of gauge invariant Hamiltonians. This is a universal way whereby one obtains candidates for classical integrable systems out of dynamical $r$-matrices. (For particular $r$-matrices some other constraints could kill the derivative term in (2.10), too.) As with constant $r$-matrices, not all these systems are necessarily Liouville integrable, and one may need to search for specific symplectic leaves in the reduced phase space to obtain interesting examples. But all these systems are at least 'Lax integrable', since the PBs of $L$ with the Hamiltonian

$$
H(q, p, \xi)=h(L(q, p, \xi))
$$

for a $\mathcal{G}$-invariant function $h$ on $\mathcal{G}$ take the following quasi-Lax form:

$$
\{L, H\}=[R V, L]-\left(\nabla_{\xi_{\mathcal{K}}} R\right) V
$$

where $V$ is the $\mathcal{G}$-valued function on the phase space defined by

$$
V(q, p, \xi)=(\nabla h)(L(q, p, \xi))
$$


with the natural $\mathcal{G}$-valued 'gradient' of $h$. The crucial point is that (2.14) yields a Lax equation for $\dot{L}$ upon setting $\xi_{\mathcal{K}}=0$. The most important Hamiltonian is

$$
h(L)=\frac{1}{2}\langle L, L\rangle
$$

and after imposing (2.12) it generates the evolution equation

$$
\dot{L}=[R L, L] .
$$

In the examples considered later the operator $R(q)$ is zero on $\mathcal{K}$ and $\left(R(q)+\frac{\nu}{2}\right)$ maps $\mathcal{K}^{\perp}$ to $\mathcal{K}^{\perp}$ in an invertible manner. Assuming that $R(q)$ vanishes on $\mathcal{K},(2.16)$ gives rise to

$$
H(q, p, \xi)=\frac{1}{2}\langle p, p\rangle-\frac{1}{2}\left\langle\xi_{\perp}, R^{2}(q) \xi_{\perp}\right\rangle-\frac{\nu}{2}\left\langle p, \xi_{\mathcal{K}}\right\rangle+\frac{\nu^{2}}{8}\langle\xi, \xi\rangle .
$$

This Hamiltonian is of the spin Calogero type since $R(q)$ is actually either a rational or hyperbolic function of $q$. The fourth term in (2.18) is a Casimir function and the third one disappears by (2.12). The corresponding evolution equation can be obtained directly as

$$
\begin{aligned}
\dot{\xi}_{\mathcal{K}} & =\left\{\xi_{\mathcal{K}}, H\right\}=0, \\
\dot{q} & =\{q, H\}=p-\frac{\nu}{2} \xi_{\mathcal{K}}, \\
\dot{p} & =\{p, H\}=-\left\langle R(q) \xi_{\perp},(\nabla R)(q) \xi_{\perp}\right\rangle, \\
\dot{\xi}_{\perp} & =\left\{\xi_{\perp}, H\right\}=\left[\xi, \frac{\nu}{2} p+R(q)^{2} \xi_{\perp}\right] .
\end{aligned}
$$

One sees from (2.9) that if $\left(R(q)+\frac{\nu}{2}\right)$ is invertible on $\mathcal{K}^{\perp}$, then the last two equations become equivalent to (2.17) upon setting $\xi_{\mathcal{K}}=0$. We impose this constraint and are only interested in the time evolution of the quantities that are invariant under the gauge transformations operating as

$$
\left(q, p, \xi_{\perp}, L\right) \mapsto\left(q, p, e^{\kappa} \xi_{\perp} e^{-\kappa}, e^{\kappa} L e^{-\kappa}\right),
$$

where $\kappa$ is an arbitrary $\mathcal{K}$-valued function. The transformation rule of $L$ follows from the integrated version of (2.3), where in the notation we assumed that $\mathcal{G}$ is a matrix Lie algebra. The time evolution of the gauge invariant functions of $L$ is not changed if one replaces $(2.17)$ by

$$
\dot{L}=[R L-\kappa, L]
$$

for any $\mathcal{K}$-valued function $\kappa$ on the (constrained) phase space. This will be used in exhibiting the geometric meaning of the integrable systems covered by the above formalism.

Natural analogues of Proposition 1 hold also for spectral parameter dependent as well as for spectral parameter independent dynamical $r$-matrices defined [21] on an arbitrary subalgebra $\mathcal{K} \subset \mathcal{G}$. Formula (2.9) remains essentially the same for all cases, the derivative $\nabla_{\xi_{\mathcal{K}}}$ in $(2.10)$ is replaced by $\nabla_{\chi}$ with $\chi(q, p, \xi)=[q, p]+\xi_{\mathcal{K}}$ for an arbitrary self-dual $\mathcal{K}[23$. Direct verification is equally easy in any case as in (2.11). The (spectral parameter dependent variant of the) basic formula (2.10) first appeared in [17, 18, without referring to the construction of $L$ out of $R$. 
The universal character of the relationship between (2.9), (2.10) and the CDYBE was explained in [22, 23] as part of a general theory based on Lie algebroids. To be more precise, 22] deals with spectral parameter dependent and 23] with spectral parameter independent dynamical $r$ matrices. After realizing (2.10), the construction of integrable systems by imposing constraints arises immediately and can be found in all the references mentioned.

\section{Interpretation as projection of geodesic motion}

We here show that the time development of the generalized spin Calogero model defined by the Lax equation (2.17) can be obtained as a projection of the free geodesic motion on a connected Lie group $G$ with Lie algebra $\mathcal{G}$ if the $r$-matrix is quasi-triangular $(\nu=1)$, or on the Lie algebra $\mathcal{G}$ if the $r$-matrix is triangular $(\nu=0)$. In special cases this statement is already known [5, 28, 29], but these papers do not explain the relationship to dynamical $r$-matrices. Our result given by Proposition 2 below applies generally, its simple proof sheds light on the geometric origin of the Lax operators (2.9) and also directly leads to an algorithm to solve the model.

Our assumptions on $R$ are that it is compatible with the decomposition (2.1), vanishes on $\mathcal{K}$ (which can be achieved acting by a gauge transformation on the solutions of the CDYBE) and is non-degenerate in the sense that the restriction of $\left(R(q)+\frac{\nu}{2}\right)$ to $\mathcal{K}^{\perp}$ is an invertible operator. We saw that these assumptions ensure the equivalence between (2.17) and (2.19) with $\xi_{\mathcal{K}}=0$. In the triangular case one has the $r$-matrices

$$
R: \check{\mathcal{K}} \rightarrow \operatorname{End}(\mathcal{G}), \quad q \mapsto R(q):= \begin{cases}0 & \text { on } \mathcal{K}, \\ \left(\left.\operatorname{ad}_{q}\right|_{\mathcal{K}^{\perp}}\right)^{-1} & \text { on } \mathcal{K}^{\perp},\end{cases}
$$

where the inverse exists on a dense domain, e.g., if $\mathcal{G}$ is semisimple and $\mathcal{K}$ is a Cartan subalgebra. The quasi-triangular $r$-matrices satisfying our assumptions all have the form $R=R^{\theta}$ with

$$
R^{\theta}: \check{\mathcal{K}} \rightarrow \operatorname{End}(\mathcal{G}), \quad q \mapsto R^{\theta}(q):= \begin{cases}0 & \text { on } \mathcal{K}, \\ \frac{1}{2}\left(\left.\theta e^{\operatorname{ad}_{q}}\right|_{\mathcal{K}^{\perp}}+1\right)\left(\left.\theta e^{\operatorname{ad}_{q}}\right|_{\mathcal{K}^{\perp}}-1\right)^{-1} & \text { on } \mathcal{K}^{\perp},\end{cases}
$$

where $\theta$ is an automorphism of $\mathcal{G}$ that preserves also the scalar product, $\mathcal{K}$ lies in the fixpoint set of $\theta$, and the inverse that occurs is well-defined for a non-empty open subset $\check{\mathcal{K}} \subset \mathcal{K}$. These quasi-triangular dynamical $r$-matrices are due to Alekseev and Meinrenken [27] (see also [34]). Their uniqueness under the above mentioned conditions is a new result proved in Appendix A. For convenience, we introduce the notation

$$
R_{+}^{\theta}(q):=R^{\theta}(q)+\frac{1}{2}= \begin{cases}\frac{1}{2} & \text { on } \mathcal{K} \\ \left(1-\left.\theta^{-1} e^{-\operatorname{ad}_{q}}\right|_{\mathcal{K}^{\perp}}\right)^{-1} & \text { on } \mathcal{K}^{\perp}\end{cases}
$$

To study the quasi-triangular case, we take a connected Lie group $G$ with Lie algebra $\mathcal{G}$ and suppose that $\theta$ lifts to an automorphism $\Theta$ of $G$ (or work locally where such a lift exists). We

restrict the considerations to an open submanifold $\breve{G} \subset G$ of 'regular' elements $g$ that admit a 'generalized polar decomposition':

$$
g=\Theta^{-1}(\rho) e^{q} \rho^{-1} \quad \text { with } \quad \rho \in G, \quad q \in \check{\mathcal{K}} .
$$


A geodesic in $G$ is a curve $g(t)$ subject to the equation

$$
\frac{d}{d t}\left(g(t)^{-1} \dot{g}(t)\right)=0
$$

where dot also denotes 'time' derivative. We are only interested in geodesics that lie in $\check{G}$.

Proposition 2. Consider a curve of the product form

$$
g(t)=\Theta^{-1}(\rho(t)) e^{q(t)} \rho(t)^{-1}
$$

with smooth functions $q(t) \in \check{\mathcal{K}}$ and $\rho(t) \in G$. Define the 'spin' variable $\xi_{\perp}(t)$ by

$$
\xi_{\perp}(t):=R_{+}^{\theta}(q(t))^{-2} M_{\perp}(t) \in \mathcal{K}^{\perp} \quad \text { with } \quad M=M_{\mathcal{K}}+M_{\perp}:=\rho^{-1} \dot{\rho} .
$$

Then the geodesic equation (3.5) implies the same time development for the gauge invariant functions of $q, \dot{q}$ and $\xi_{\perp}$ as does the Lax equation 2.17) together with $p=\dot{q}$.

We start the proof by noticing that

$$
g^{-1} \dot{g}=\rho\left(\dot{q}-\left(1-\theta^{-1} e^{-\operatorname{ad}_{q}}\right) M\right) \rho^{-1}=\rho\left(\dot{q}-\left(1-\left.\theta^{-1} e^{-\operatorname{ad}_{q}}\right|_{\mathcal{K}^{\perp}}\right) M_{\perp}\right) \rho^{-1} .
$$

By using the definitions in (3.3) and (3.7), this is equivalently written as

$$
g^{-1} \dot{g}=\rho\left(\dot{q}-R_{+}^{\theta}(q) \xi_{\perp}\right) \rho^{-1}=\rho L \rho^{-1}
$$

in terms of the Lax operator

$$
L\left(q, \dot{q}, \xi_{\perp}\right)=\dot{q}-R_{+}^{\theta}(q) \xi_{\perp},
$$

which has the same form as (2.9) with $\dot{q}=p$ and $\xi_{\mathcal{K}}=0$. Continuing with (3.9), (3.5) takes the form

$$
0=\frac{\mathrm{d}}{\mathrm{d} t}\left(g(t)^{-1} \dot{g}(t)\right)=\rho(\dot{L}-[L, M]) \rho^{-1} .
$$

Since $M=M_{\mathcal{K}}+R_{+}^{\theta}(q)^{2} \xi_{\perp}$, this in turn is equivalent to

$$
\dot{L}=\left[L, R_{+}^{\theta}(q)^{2} \xi_{\perp}+M_{\mathcal{K}}\right] .
$$

The 'geodesic Lax equation' (3.12) is to be compared with the 'spin Calogero Lax equation' (2.17), which now reads

$$
\dot{L}=\left[R^{\theta}(q) L, L\right]=\left[R_{+}^{\theta}(q) L, L\right]=\left[L, R_{+}^{\theta}(q)^{2} \xi_{\perp}-\frac{1}{2} \dot{q}\right]
$$

The difference of the evolutional derivatives of $L$ defined by (3.12) and by (3.13) is an infinitesimal gauge transformation in the sense of (2.21), and thus the proposition follows.

It is worth remarking that originally the gauge transformations (2.20) arose from the first class constraints (2.12), but now we can also view them as due to the obvious ambiguity of the parametrization of $g$ in (3.4). It is not difficult to show that this parametrization is applicable 
in a neighbourhood of $e^{q_{0}} \in G$ if and only if the $r$-matrix (3.2) is regular at $q_{0} \in \mathcal{K}$. In fact, for any such $q_{0} \in \mathcal{K}$ the elements of $G$ in a neighbourhood of $e^{q_{0}}$ can be uniquely written as ${ }^{1}$

$$
g=\Theta^{-1}\left(e^{\eta}\right) e^{q} e^{-\eta}
$$

where $q$ varies in $\mathcal{K}$ around $q_{0}$ and $\eta$ varies in $\mathcal{K}^{\perp}$ around zero. By combining this with Proposition 2 one can in principle solve the spin Calogero equation of motion (for gauge invariant quantities) as follows. First, take an initial value $\left(q_{0}, p_{0}=\dot{q}_{0},\left(\xi_{\perp}\right)_{0}\right)$. Next, construct $L_{0}$ out of these data according to (3.10). Set $\rho_{0}:=e \in G$, and thus obtain from (3.5), (3.9) the geodesic

$$
g(t)=e^{q_{0}} e^{t L_{0}} .
$$

Then, decompose this geodesic according to (3.14) in the form

$$
g(t)=\Theta^{-1}(\rho(t)) e^{q(t)} \rho^{-1}(t) \quad \text { with } \quad \rho(t)=e^{\eta(t)}, \quad \eta(t) \in \mathcal{K}^{\perp},
$$

which is possible at least for small values of $t$. Finally, determine $\xi_{\perp}(t)$ from (3.7) and find also $L(t)$ from (3.9) . This procedure provides the solution of the spin Calogero equation of motion (constrained by $\xi_{\mathcal{K}}=0$ ) up to a time dependent gauge transformation of the type in (2.20), but this is enough since we are interested only in gauge invariant quantities.

The non-degenerate rational $r$-matrices (3.1) are related to geodesic motion on the Lie algebra $\mathcal{G}$. To see this let us consider a curve $X(t) \in \mathcal{G}$ having the product form

$$
X(t)=\rho(t) q(t) \rho(t)^{-1}, \quad \rho(t) \in G, \quad q(t) \in \check{\mathcal{K}} .
$$

This gives rise to $\dot{X}=\rho L \rho^{-1}$, where $R(q)$ is given by (3.1) and

$$
L=\dot{q}-R(q) \xi_{\perp} \quad \text { with } \quad \xi_{\perp}=R(q)^{-2} M_{\perp}, \quad M=M_{\mathcal{K}}+M_{\perp}=\rho^{-1} \dot{\rho} .
$$

Then the equation of motion $\frac{d^{2} X(t)}{d t^{2}}=0$ translates into the 'geodesic Lax equation'

$$
\dot{L}=[L, M]=\left[R(q) L-M_{\mathcal{K}}, L\right] .
$$

This is gauge equivalent to the 'spin Calogero Lax equation' (2.17) associated with the 'principal rational $r$-matrix' (3.1) by the construction in Section 2.

The solution algorithm sketched above must be equivalent to the one in [23] for the overlap of the cases considered (which actually include only the systems (5.4)). Our derivation by the projection method was inspired by the seminal work of Olshanetsky and Perelomov [1] and it appears much simpler to us than the derivation found in Section 6 of [23].

For a simple Lie group $G$ and $\theta=$ id our Proposition 2 is consistent with Reshetikhin's identification [28] of the 'principal trigonometric' spin Calogero model (with Hamiltonian of the form in (5.4) or (5.8) below) as the result of factoring the natural Hamiltonian system on $T^{*} \check{G}$ by the adjoint action of $G$. Here $\breve{G}$ is the set regular elements in the usual sense, and $q$ (restricted to an open Weyl alcove) together with its momentum $p$ and the spin variable in $\mathcal{K}^{\perp} / e^{\mathcal{K}}$ provide coordinates on the factor space $T^{*} \check{G} / G$. Next we generalize this result to arbitrary automorphisms.

\footnotetext{
${ }^{1}$ One sees this by calculating the derivative of the map $m: \mathcal{K} \times \mathcal{K}^{\perp} \ni(q, \eta) \mapsto \Theta^{-1}\left(e^{\eta}\right) e^{q} e^{-\eta} \in G$ at $\left(q_{0}, 0\right)$.
} 


\section{$4 \quad$ Reduction of $T^{*} G$ by twisted conjugations}

We have seen that the spin Calogero equation of motion (3.13) results from the geodesic equation on the Lie group $G$. The geodesic motion corresponds to the Hamiltonian system $\left(T^{*} G, \Omega, H\right)$ with the natural symplectic form $\Omega$ and 'kinetic energy' $H$ associated with $\langle$,$\rangle . In this section$ we demonstrate that the spin Calogero phase space together with its Poisson structure and Hamiltonian can be identified as a Hamiltonian reduction of $\left(T^{*} G, \Omega, H\right)$.

As might be guessed from (3.6) and the result of [28] for $\Theta=$ id, the reduction is by the cotangent lift of the 'twisted adjoint action' of $G$ associated with $\Theta$. We trivialize $T^{*} G$ by right translations and identify $\mathcal{G}^{*}$ with $\mathcal{G}$ with the aid of the scalar product. This gives the model

$$
T^{*} G \simeq G \times \mathcal{G}=\{(g, J) \mid g \in G, \quad J \in \mathcal{G}\}
$$

with

$$
\Omega=d\left\langle J,(d g) g^{-1}\right\rangle, \quad H=\frac{1}{2}\langle J, J\rangle .
$$

We then consider the $\Theta$-twisted adjoint action defined by

$$
\operatorname{Ad}_{k}^{\Theta}:(g, J) \mapsto\left(\Theta^{-1}(k) g k^{-1}, \Theta^{-1}(k) J \Theta^{-1}\left(k^{-1}\right)\right) \quad \forall k \in G,
$$

where our notation is adapted to matrix Lie algebras as usual. The equivariant momentum map generating this Hamiltonian action of $G, \psi: T^{*} G \rightarrow \mathcal{G}^{*} \simeq \mathcal{G}$, is readily found

$$
\psi:(g, J) \mapsto \theta(J)-g^{-1} J g .
$$

Our definition in (4.3) may look slightly cumbersome as far as the usage of the inverse of $\Theta$ is concerned, but this is how it fits with the form of the $r$-matrix chosen in (3.2).

We are interested in the Marsden-Weinstein reduced phase $\operatorname{spaces}^{2}\left(T^{*} G\right)_{\psi=\mu} / G_{\mu}$, where $\mu \in$ $\mathcal{G}^{*} \cap \psi\left(T^{*} G\right)$ is chosen arbitrarily and $G_{\mu} \subset G$ is its isotropy group. We recall that $\left(T^{*} G\right)_{\psi=\mu} / G_{\mu}$ carries a non-degenerate Poisson structure induced by the projection of the symplectic form $\Omega$. These reduced phase spaces together encode the structure of $T^{*} G / G$, since they correspond to the symplectic leaves (or unions of leaves) in $T^{*} G / G$ in a one-to-one manner, simply by means of projecting $\left(T^{*} G\right)_{\psi=\mu} \subset T^{*} G$ to the $G$-orbits passing through it. For a textbook, see e.g. [37].

Let $\mathcal{O}$ denote the coadjoint orbit of $G$ through $-\mu$. It will be convenient to realize $\left(T^{*} G\right)_{\psi=\mu} / G_{\mu}$ by using the standard 'shifting trick' as

$$
\left(T^{*} G\right)_{\psi=\mu} / G_{\mu} \simeq\left(T^{*} G \times \mathcal{O}\right)_{\tilde{\psi}=0} / G
$$

where $\mathcal{O}$ is equipped with its own symplectic form, $\omega^{\mathcal{O}}$, and $\tilde{\psi}$ is the momentum map for the diagonal action, $\widetilde{\operatorname{Ad}}_{k}^{\Theta}$, of $G$ on $T^{*} G \times \mathcal{O}$ :

$$
\widetilde{\operatorname{Ad}}_{k}^{\Theta}: T^{*} G \times \mathcal{O} \ni(g, J, \xi) \mapsto\left(\Theta^{-1}(k) g k^{-1}, \Theta^{-1}(k) J \Theta^{-1}\left(k^{-1}\right), k \xi k^{-1}\right), \quad \forall k \in G .
$$

\footnotetext{
${ }^{2}$ The factor spaces that appear here are not everywhere smooth manifolds, but possess some singularities. This complication could be resolved, e.g., by restriction to a dense and open smooth component (the principal isotropy type for the group action) or by invoking the theory of stratified symplectic spaces and singular reduction [35, 36. In this paper we disregard these technicalities, since they do not affect our main point.
} 
The symplectic form on $T^{*} G \times \mathcal{O}$ is given by

$$
\Omega^{\mathcal{O}}=\Omega+\omega^{\mathcal{O}}
$$

and the associated momentum map reads

$$
\tilde{\psi}(g, J, \xi)=\theta(J)-g^{-1} J g+\xi .
$$

The identification (4.5) means that the reduced symplectic structure can be induced from $\Omega^{\mathcal{O}}$.

We wish to show that the symplectic leaves of the spin Calogero phase space defined by the construction of [22, 23] outlined in Section 2 fill certain dense subsets in the MarsdenWeinstein reduced phase spaces (4.5). The appropriate symplectic leaves result by performing the construction of Section 2 in such a way that the spin variable is restricted there to the orbit $\mathcal{O}$ from the beginning. To avoid notational complications, here we make the simplifying assumption that $G$ is a compact, connected simple Lie group. Denote by $G^{\Theta}$ the set of fixpoints of the automorphism $\Theta$ of $G$. In the compact case the generic orbits in $G$ under the $\Theta$-twisted adjoint action, which we call twisted conjugacy classes, can be parametrized by a certain alcove in a maximal torus $K$ of $G^{\Theta}$ (see [38] and references therein). It is also known [39] that $\left(1-\theta^{-1} e^{-\operatorname{ad}_{q}}\right)$ is invertible on $\mathcal{K}^{\perp}$ for generic elements $q \in \mathcal{K}$, where $\mathcal{K}$ is the Lie algebra of $K$.

Proposition 3. Consider an automorphism $\Theta$ of a compact, connected simple Lie group $G$ and a Cartan subalgebra $\mathcal{K}$ of the Lie algebra of $G^{\Theta}$. Let $\check{G} \subset G$ be the $\operatorname{Ad}_{G}^{\Theta}$-invariant open submanifold that consists of the $\Theta$-twisted regular conjugacy classes represented by $e^{q}$ with $q \in \check{\mathcal{K}}$, where the operator $\left(1-\theta^{-1} e^{-\mathrm{ad}_{q}}\right) \mid \mathcal{K}^{\perp}$ is invertible and $q \mapsto e^{q}$ is injective on the open submanifold $\check{\mathcal{K}} \subset \mathcal{K}$. Take an arbitrary $\mu \in \psi\left(T^{*} \check{G}\right)$ and denote by $\mathcal{O}$ the coadjoint orbit of $G$ through $-\mu$. Then the Marsden-Weinstein reduced phase space $\left(T^{*} \check{G} \times \mathcal{O}\right)_{\tilde{\psi}=0} / G$ (see 4.5)) is identical to the phase space of the spin Calogero system defined in Section 2 by imposing the first class constraint (2.12) on $T^{*} \check{\mathcal{K}} \times \mathcal{O} \subset \mathcal{M}$ (2.7).

To prove Proposition 3, the key point is to solve the momentum map constraint $\tilde{\psi}=0$ (4.8) by first bringing $g \in \check{G}$ to its 'diagonal' representative $e^{q}$ with $q \in \check{\mathcal{K}}$. After doing this, the constraint $\tilde{\psi}=0$ becomes

$$
\theta(J)-e^{-\operatorname{ad}_{q}}(J)+\xi=0,
$$

which is easily seen to be equivalent to

$$
\xi_{\mathcal{K}}=0 \quad \text { and } \quad \theta(J)=J_{\mathcal{K}}-R_{+}^{\theta}(q) \xi_{\perp},
$$

where $R_{+}^{\theta}(q)$ is defined in (3.3). The resulting manifold, $S \subset\left(T^{*} \check{G} \times \mathcal{O}\right)_{\tilde{\psi}=0}$ given by

$$
S:=\left\{\left(e^{q}, J_{\mathcal{K}}-\theta^{-1} R_{+}^{\theta}(q) \xi_{\perp}, \xi_{\perp}\right) \mid q \in \check{\mathcal{K}}, J_{\mathcal{K}} \in \mathcal{K}, \xi_{\perp} \in \mathcal{O} \cap \mathcal{K}^{\perp}\right\}
$$

is the gauge slice of a global partial gauge fixing for the action of $G$ on $\left(T^{*} \check{G} \times \mathcal{O}\right)_{\tilde{\psi}=0}$. By inspecting this partial gauge fixing one sees that

$$
\left(T^{*} \check{G} \times \mathcal{O}\right)_{\tilde{\psi}=0} / G \simeq S / K \simeq \check{\mathcal{K}} \times \mathcal{K} \times\left(\mathcal{O} \cap \mathcal{K}^{\perp}\right) / K
$$


where $K$ is the Lie subgroup of $G^{\Theta}$ corresponding to $\mathcal{K}$ and we used the identification $S \simeq$ $\check{\mathcal{K}} \times \mathcal{K} \times\left(\mathcal{O} \cap \mathcal{K}^{\perp}\right)$ defined by the parameters $\left(q, J_{\mathcal{K}}, \xi_{\perp}\right)$ on $S$. In terms of these variables the restriction of $\Omega^{\mathcal{O}}$ (4.7) to $S$ takes the following form:

$$
\left.\Omega^{\mathcal{O}}\right|_{S}=d\left\langle J_{\mathcal{K}}, d q\right\rangle+\left.\omega^{\mathcal{O}}\right|_{\mathcal{O} \cap \mathcal{K}^{\perp}}
$$

By setting $p:=J_{\mathcal{K}}$, this becomes identical to the restriction of the symplectic form of $T^{*} \check{\mathcal{K}} \times \mathcal{O}$ underlying the PBs (2.8), which is obtained by imposing the first class constraint (2.12) that generate the factorization ${ }^{3}$ by $K$. This proves Proposition 3.

Notice that upon setting $p=J_{\mathcal{K}}, \theta(J)$ in the gauge $S$ (4.11) yields precisely the constrained quasi-Lax operator (2.9). It follows that the Hamiltonian also reduces as required, since $H(g, J)=\frac{1}{2}\langle J, J\rangle=\frac{1}{2}\langle\theta(J), \theta(J)\rangle$.

Equation (4.4) implies that $\psi\left(T^{*} \check{G}\right)$ consists of the coadjoint orbits of $G$ through the elements of $\mathcal{K}^{\perp} \subset \mathcal{G}^{*}$, and it is also clear that $\left(T^{*} \check{G}\right)_{\psi=\mu}$ is dense in $\left(T^{*} G\right)_{\psi=\mu}$ for any $\mu \in \psi\left(T^{*} \check{G}\right)$. It would be interesting to further elaborate the relationship between the reduced systems arising from $T^{*} \check{G}$ and from the entire $T^{*} G$.

For more general reductive groups, the spin Calogero model constructed in Section 2 is essentially (up to a discrete symmetry) identical to the factor of $T^{*} \breve{G}$ by $G$, where $\breve{G}$ consists of the elements $g$ in (3.4) and $G$ acts by twisted conjugations. It is an open problem to explore the possible connections between the global structure of the reduction of $T^{*} G$ and the spin Calogero models for compact as well as for non-compact groups. In studying this problem one has to take into account the non-conjugate Cartan subgroups of $G^{\Theta}$ that exist in general and all $\Theta$-twisted conjugacy classes, whose description is rather complicated even for $\Theta=$ id [40].

It is easy to show that the rational spin Calogero model corresponding to the $r$-matrix (3.1) is a reduction of the geodesic system on $T^{*} \breve{\mathcal{G}}$, where $\check{\mathcal{G}} \subset \mathcal{G}$ contains the adjoint orbits of $G$ through $\check{\mathcal{K}}$. For a compact simple Lie algebra $\mathcal{G}$ with Cartan subalgebra $\mathcal{K}$ this statement was proved in [5], without mention of $r$-matrices. See also [29, 31] for similar results about rational spin Calogero models associated with Cartan involutions.

Let $I_{h}$ be an inner automorphism of $G$, operating as $I_{h}: G \ni g \mapsto h g h^{-1}$ with a fixed $h \in G$, and consider the automorphism $\tilde{\Theta}:=\Theta \circ I_{h}$. The corresponding twisted adjoint actions of $G$ on $T^{*} G$ are related by the formula

$$
\operatorname{Ad}_{k}^{\tilde{\Theta}}=\mathcal{L}_{h^{-1}} \circ \operatorname{Ad}_{k}^{\Theta} \circ \mathcal{L}_{h} \quad \forall k \in G .
$$

Here $\mathcal{L}_{h}: T^{*} G \rightarrow T^{*} G$ is the symplectomorphism $\mathcal{L}_{h}:(g, J) \mapsto\left(h g, h J h^{-1}\right)$, which also preserves the kinetic energy. It follows from (4.14) that the symmetry reductions of the geodesic system on $T^{*} G$ by the $\Theta$-twisted and the $\tilde{\Theta}$-twisted adjoint actions lead to reduced Hamiltonian systems that are isomorphic by a map induced from $\mathcal{L}_{h}$. By Proposition 3, this implies the isomorphic nature of the integrable spin Calogero models that can be obtained by applying the method of [22, 23] to 'input data' related by an inner automorphism of $\mathcal{G}$. In fact, one expects on general grounds that only different cosets in $\operatorname{Aut}(\mathcal{G}) / \operatorname{Int}(\mathcal{G})$ correspond to significantly different

\footnotetext{
${ }^{3}$ The singularities of $S / K$ can be avoided by restriction to a dense open subset of $\mathcal{O}$ in (4.12), like in [22, 23].
} 
integrable systems, and this could be confirmed directly as well without using the Hamiltonian reduction of $T^{*} G$.

\section{$5 \quad$ Examples based on involutive diagram automorphisms of real split and compact simple Lie algebras}

As an illustration, we here present the spin Calogero systems associated with a real split or a compact simple Lie algebra equipped with an involutive automorphism induced from the Dynkin diagram. The systems corresponding to the non-trivial diagram automorphisms of the $A_{n}, D_{n}$ and $E_{6}$ algebras have not been described before, while those associated with the trivial diagram automorphism appear also, e.g., in [22, 23, 25, 28]. To keep the presentation short, in all cases we write down only the $r$-matrix and the Hamiltonian after imposing the constraint $\xi_{\mathcal{K}}=0$. See eqs. (5.4), (5.8) and (5.14), (5.17) for the Hamiltonians corresponding to the trivial and non-trivial automorphisms, respectively.

First, we introduce some notations relying on standard results about Lie algebras. Let $\mathcal{A}$ be a simple complex Lie algebra with Killing form $\langle$,$\rangle , Cartan subalgebra \mathcal{H}$, set of simple roots $\Pi=\left\{\varphi_{k}\right\}$ and positive roots $\Phi_{+}$. Choose root vectors $X_{ \pm \varphi_{k}}$ normalized so that $\left\langle X_{\varphi_{k}}, X_{-\varphi_{k}}\right\rangle=1$. Any symmetry $\tau$ of the Dynkin diagram of $\mathcal{A}$ extends to an automorphism of $\Phi_{+}$as well as to an automorphism of $\mathcal{A}$ by the requirement $\tau\left(X_{ \pm \varphi_{k}}\right)=X_{ \pm \tau\left(\varphi_{k}\right)}$ for any $\varphi_{k} \in \Pi$. The diagram automorphism $\tau$ of $\mathcal{A}$ commutes with the Chevalley automorphism $\sigma$ defined by $\sigma\left(X_{ \pm \varphi_{k}}\right)=$ $-X_{\mp \varphi_{k}}$. We fix root vectors $X_{ \pm \varphi}$ in such a way that

$$
X_{-\varphi}=-\sigma\left(X_{\varphi}\right) \quad \text { and } \quad\left\langle X_{\varphi}, X_{-\varphi}\right\rangle=1 \quad \forall \varphi \in \Phi_{+} .
$$

We denote by $\mathcal{H}_{r}$ the real span of the elements $T_{\varphi_{k}}:=\left[X_{\varphi_{k}}, X_{-\varphi_{k}}\right]$.

With the above conventions, the split (normal) real form of $\mathcal{A}$ is the Lie algebra $\mathcal{A}_{r}$ defined by the real span of the base elements $T_{\varphi_{k}}$ and $X_{ \pm \varphi}$. To describe the example provided by the data $\mathcal{G}:=\mathcal{A}_{r}, \mathcal{K}:=\mathcal{H}_{r}, \theta=\mathrm{id}$, we expand the 'spin' variable $\xi \in \mathcal{A}_{r}$ as

$$
\xi=\xi_{\mathcal{H}_{r}}+\sum_{\varphi \in \Phi} \xi_{\varphi} X_{\varphi} \quad \text { with } \quad \xi_{\mathcal{H}_{r}} \in \mathcal{H}_{r}
$$

Equation (3.2) gives the standard $r$-matrix

$$
R(q) \xi=\frac{1}{2} \sum_{\varphi \in \Phi} \xi_{\varphi} \operatorname{coth} \frac{\varphi(q)}{2} X_{\varphi}
$$

where $q \in \check{\mathcal{H}}_{r}$ is such that this function is well-defined. Upon imposing the constraint $\xi_{\mathcal{H}_{r}}=0$, the spin Calogero Hamiltonian (2.18) takes the form

$$
H(q, p, \xi)=\frac{1}{2}\langle p, p\rangle-\frac{1}{4} \sum_{\varphi \in \Phi_{+}} \frac{\xi_{\varphi} \xi_{-\varphi}}{\sinh ^{2} \frac{\varphi(q)}{2}} .
$$


The compact real form $\mathcal{A}_{c}$ of $\mathcal{A}$ is the real span of the base elements iT $T_{\varphi_{k}}$ together with

$$
Y_{\varphi}:=\frac{\mathrm{i}}{\sqrt{2}}\left(X_{\varphi}+X_{-\varphi}\right) \quad \text { and } \quad Z_{\varphi}:=\frac{1}{\sqrt{2}}\left(X_{\varphi}-X_{-\varphi}\right) \quad \forall \varphi \in \Phi_{+} .
$$

For $\mathcal{G}:=\mathcal{A}_{c}, \mathcal{K}:=\mathrm{i} \mathcal{H}_{r}$ and $\theta=\mathrm{id}$ using the expansion

$$
\xi=\mathrm{i} \xi_{\mathcal{H}_{r}}+\sum_{\varphi \in \Phi_{+}}\left(\eta_{\varphi} Y_{\varphi}+\zeta_{\varphi} Z_{\varphi}\right)
$$

we have

$$
R(\mathrm{i} q) \xi=\frac{1}{2} \sum_{\varphi \in \Phi_{+}} \cot \frac{\varphi(q)}{2}\left(\eta_{\varphi} Z_{\varphi}-\zeta_{\varphi} Y_{\varphi}\right) .
$$

In this case the spin Calogero variables are $(\mathrm{i} q, \mathrm{i} p, \xi) \in \check{\mathcal{K}} \times \mathcal{K} \times \mathcal{K}^{\perp}$, and the Hamiltonian reads

$$
H(\mathrm{i} q, \mathrm{i} p, \xi)=-\frac{1}{2}\langle p, p\rangle-\frac{1}{8} \sum_{\varphi \in \Phi_{+}} \frac{\eta_{\varphi}^{2}+\zeta_{\varphi}^{2}}{\sin ^{2} \frac{\varphi(q)}{2}},
$$

where $q \in \mathcal{H}_{r}$ is such that $\sin \frac{\varphi(q)}{2} \neq 0$.

Now let us consider an involutive automorphism $\tau$ of $\mathcal{A}$ induced from a Dynkin diagram automorphism as outlined above. Notice that $\tau$ preserves the split and the compact real forms as well as the real Cartan subalgebra $\mathcal{H}_{r}$. Denote by $\mathcal{A}^{ \pm}, \mathcal{A}_{r}^{ \pm}, \mathcal{A}_{c}^{ \pm}, \mathcal{H}^{ \pm}$and $\mathcal{H}_{r}^{ \pm}$the corresponding eigensubspaces of $\tau$ with eigenvalues \pm 1 . It is known that $\mathcal{A}^{+}$is a complex simple Lie algebra with Cartan subalgebra $\mathcal{H}^{+}$, and $\mathcal{A}^{-}$is an irreducible module of $\mathcal{A}^{+}$whose non-zero weights have multiplicity one 39]. Furthermore, $\left(\mathcal{H}_{r}^{+}, \mathcal{A}_{r}^{+}\right)$and $\left(\mathrm{i} \mathcal{H}_{r}^{+}, \mathcal{A}_{c}^{+}\right)$are the split and compact real forms of $\left(\mathcal{H}^{+}, \mathcal{A}^{+}\right)$with the associated real irreducible modules $\mathcal{A}_{r}^{-}$and $\mathcal{A}_{c}^{-}$, respectively. Convenient bases of all these spaces can be obtained from the above considered ('re-normalized') Chevalley basis of $\mathcal{A}$ by the 'folding' procedure. We recall this in Appendix $\mathrm{B}$, and next summarize the result only.

Let $\Delta$ be the set of roots of $\left(\mathcal{H}^{+}, \mathcal{A}^{+}\right)$with associated root vectors $X_{\alpha}^{+}$for $\alpha \in \Delta$. Let $\Gamma$ denote the set of non-zero weights for $\left(\mathcal{H}^{+}, \mathcal{A}^{-}\right)$with corresponding weight vectors $X_{\lambda}^{-}$for $\lambda \in \Gamma$. We can choose these elements so that

$$
\left\langle X_{\alpha}^{+}, X_{-\alpha}^{+}\right\rangle=1=\left\langle X_{\lambda}^{-}, X_{-\lambda}^{-}\right\rangle \quad \text { and } \quad \sigma\left(X_{\alpha}^{+}\right)=-X_{-\alpha}^{+}, \quad \sigma\left(X_{\lambda}^{-}\right)=-X_{-\lambda}^{-} .
$$

The folding procedure ensures that these bases are such that $\mathcal{A}_{r}^{+}$is the real span of $\mathcal{H}_{r}^{+}$together with the $X_{\alpha}^{+}$for $\alpha \in \Delta$, and $\mathcal{A}_{r}^{-}$is spanned by $\mathcal{H}_{r}^{-}$and the $X_{\lambda}^{-}$for $\lambda \in \Gamma$. Let $\Delta_{+}$and $\Gamma_{+}$ denote positive roots and weights. The folding procedure also ensures that $\mathcal{A}_{c}^{+}$is the real span of $i \mathcal{H}_{r}^{+}$together with basis vectors

$$
Y_{\alpha}^{+}:=\frac{\mathrm{i}}{\sqrt{2}}\left(X_{\alpha}^{+}+X_{-\alpha}^{+}\right) \quad \text { and } \quad Z_{\alpha}^{+}:=\frac{1}{\sqrt{2}}\left(X_{\alpha}^{+}-X_{-\alpha}^{+}\right) \quad \forall \alpha \in \Delta_{+},
$$

and $\mathcal{A}_{c}^{-}$is the real span of $\mathrm{i} \mathcal{H}_{r}^{-}$together with basis vectors

$$
Y_{\lambda}^{-}:=\frac{\mathrm{i}}{\sqrt{2}}\left(X_{\lambda}^{-}+X_{-\lambda}^{-}\right) \quad \text { and } \quad Z_{\lambda}^{-}:=\frac{1}{\sqrt{2}}\left(X_{\lambda}^{-}-X_{-\lambda}^{-}\right) \quad \forall \lambda \in \Gamma_{+} .
$$


Now we consider the system associated with the data $\mathcal{G}:=\mathcal{A}_{r}, \mathcal{K}:=\mathcal{H}_{r}^{+}, \theta:=\tau$. We write $\xi \in \mathcal{A}_{r}$ as

$$
\xi=\xi_{\mathcal{H}_{r}}^{+}+\xi_{\mathcal{H}_{r}}^{-}+\sum_{\alpha \in \Delta} \xi_{\alpha}^{+} X_{\alpha}^{+}+\sum_{\lambda \in \Gamma} \xi_{\lambda}^{-} X_{\lambda}^{-} \quad \text { with } \quad \xi_{\mathcal{H}_{r}}^{ \pm} \in \mathcal{H}_{r}^{ \pm}
$$

According to (3.2)

$$
R^{\tau}(q) \xi=\frac{1}{2} \sum_{\alpha \in \Phi} \xi_{\alpha}^{+} \operatorname{coth} \frac{\alpha(q)}{2} X_{\alpha}^{+}+\frac{1}{2} \sum_{\lambda \in \Gamma} \xi_{\lambda}^{-} \tanh \frac{\lambda(q)}{2} X_{\lambda}^{-},
$$

where this function is smooth for $q \in \check{\mathcal{H}}_{r}^{+}$. After imposing the constraint $\xi_{\mathcal{H}_{r}}^{+}=0$, we obtain from (2.18) the Hamiltonian

$$
H(q, p, \xi)=\frac{1}{2}\langle p, p\rangle-\frac{1}{4} \sum_{\alpha \in \Delta_{+}} \frac{\xi_{\alpha}^{+} \xi_{-\alpha}^{+}}{\sinh ^{2} \frac{\alpha(q)}{2}}+\frac{1}{8}\left\langle\xi_{\mathcal{H}_{r}}^{-}, \xi_{\mathcal{H}_{r}}^{-}\right\rangle+\frac{1}{4} \sum_{\lambda \in \Gamma_{+}} \frac{\xi_{\lambda}^{-} \xi_{-\lambda}^{-}}{\cosh ^{2} \frac{\lambda(q)}{2}}
$$

In the corresponding compact case, with data $\mathcal{G}:=\mathcal{A}_{c}, \mathcal{K}:=\mathrm{i} \mathcal{H}_{r}^{+}, \theta=\tau$, we parametrize $\xi \in \mathcal{A}_{c}$ as

$$
\xi=\mathrm{i} \xi_{\mathcal{H}_{r}}^{+}+\mathrm{i} \xi_{\mathcal{H}_{r}}^{-}+\sum_{\alpha \in \Delta_{+}}\left(\eta_{\alpha}^{+} Y_{\alpha}^{+}+\zeta_{\alpha}^{+} Z_{\alpha}^{+}\right)+\sum_{\lambda \in \Gamma_{+}}\left(\eta_{\lambda}^{-} Y_{\lambda}^{-}+\zeta_{\lambda}^{-} Z_{\lambda}^{-}\right)
$$

For i $q \in \check{\mathcal{K}} \subset \mathrm{i} \mathcal{H}_{r}^{+}$, we then obtain

$$
R^{\tau}(\mathrm{i} q) \xi=\frac{1}{2} \sum_{\alpha \in \Delta_{+}} \cot \frac{\alpha(q)}{2}\left(\eta_{\alpha}^{+} Z_{\alpha}^{+}-\zeta_{\alpha}^{+} Y_{\alpha}^{+}\right)-\frac{1}{2} \sum_{\lambda \in \Gamma_{+}} \tan \frac{\lambda(q)}{2}\left(\eta_{\lambda}^{-} Z_{\lambda}^{-}-\zeta_{\lambda}^{-} Y_{\lambda}^{-}\right)
$$

After imposing the first class constraints $\xi_{\mathcal{H}_{r}}^{+}=0$, the spin Calogero variables are (i $q$, i $\left.p, \xi\right) \in$ $\check{\mathcal{K}} \times \mathcal{K} \times \mathcal{K}^{\perp}$, and the Hamiltonian reads

$$
H(\mathrm{i} q, \mathrm{i} p, \xi)=-\frac{1}{2}\langle p, p\rangle-\frac{1}{8} \sum_{\alpha \in \Delta_{+}} \frac{\left(\eta_{\alpha}^{+}\right)^{2}+\left(\zeta_{\alpha}^{+}\right)^{2}}{\sin ^{2} \frac{\alpha(q)}{2}}-\frac{1}{8}\left\langle\xi_{\mathcal{H}_{r}}^{-}, \xi_{\mathcal{H}_{r}}^{-}\right\rangle+\frac{1}{8} \sum_{\lambda \in \Gamma_{+}} \frac{\left(\eta_{\lambda}^{-}\right)^{2}+\left(\zeta_{\lambda}^{-}\right)^{2}}{\cos ^{2} \frac{\lambda(q)}{2}}
$$

We finish by listing the positive roots $\Delta_{+}$and weights $\Gamma_{+}$that arise for the involutive diagram automorphisms of the classical Lie algebras.

If $\mathcal{A}=D_{n+1}$, then $\mathcal{A}^{+}=B_{n}$ and the module $\mathcal{A}^{-}$is isomorphic to the defining representation of $B_{n}$. Therefore

$$
\Delta_{+}=\left\{e_{k} \pm e_{l}, e_{m} \mid 1 \leq k<l \leq n, 1 \leq m \leq n\right\}, \quad \Gamma_{+}=\left\{e_{m} \mid 1 \leq m \leq n\right\}
$$

One may realize $\mathcal{H}_{r}^{+}$as the space of real diagonal matrixes, $q$, of the form

$$
q=\operatorname{diag}\left(q_{1}, \ldots, q_{n}, 0,0,-q_{n}, \ldots,-q_{1}\right) \quad \text { and } \quad e_{m}: q \mapsto q_{m} .
$$

If $\mathcal{A}=A_{2 n-1}$, then $\mathcal{A}^{+}=C_{n}$ and

$$
\Delta_{+}=\left\{e_{k} \pm e_{l}, 2 e_{m} \mid 1 \leq k<l \leq n, 1 \leq m \leq n\right\}, \quad \Gamma_{+}=\left\{e_{k} \pm e_{l} \mid 1 \leq k<l \leq n\right\} .
$$


Now $\mathcal{H}_{r}^{+}$can be realized as the space of real diagonal matrixes written as

$$
q=\operatorname{diag}\left(q_{1}, \ldots, q_{n},-q_{n}, \ldots,-q_{1}\right) \quad \text { and } \quad e_{m}: q \mapsto q_{m}
$$

For $\mathcal{A}=A_{2 n}$ one has $\mathcal{A}^{+}=B_{n}$ with $\Delta_{+}$as in (5.18) and

$$
\Gamma_{+}=\left\{e_{k} \pm e_{l}, e_{m}, 2 e_{m} \mid 1 \leq k<l \leq n, 1 \leq m \leq n\right\}
$$

Now $\mathcal{H}_{r}^{+}$consists of the real diagonal matrices

$$
q=\operatorname{diag}\left(q_{1}, \ldots, q_{n}, 0,-q_{n}, \ldots,-q_{1}\right) \quad \text { and } \quad e_{m}: q \mapsto q_{m}
$$

In the $\mathcal{A}=E_{6}$ case $\mathcal{A}^{+}=F_{4}$ and the weights of its representation on $\mathcal{A}^{-}$can be found in [39]. Of course, one can also construct an integrable spin Calogero model by using the third order diagram automorphism of $D_{4}$.

If in the above we allow all variables to be complex, then the models given by (5.4) and (5.8) (or respectively by (5.14) and (5.17) ) yield the holomorphic spin Calogero model belonging to the complex Lie algebra $\mathcal{A}$ equipped with the corresponding automorphism. In other words, we obtained different real forms of complex spin Calogero models using the real forms $\mathcal{A}_{r}$ and $\mathcal{A}_{c}$ of $\mathcal{A}$. For general considerations about real forms of complexified Hamiltonian systems with the spinless Calogero models as examples, we refer to [41].

\section{Discussion}

One of the original motivations behind the theory of constant and dynamical classical $r$-matrices is the potential application to build integrable systems using the $r$-matrices. The aim of the present paper has been to contribute to this theory by characterizing the systems that correspond by the construction of [22, 23] to the non-degenerate dynamical $r$-matrices (3.2). (See also the uniqueness statement of Proposition A.2 in Appendix A.) We demonstrated that these spin Calogero type models are projections of the natural geodesic system on the underlying Lie group $G$, where the automorphism enters through the twisted adjoint action of $G$. The Hamiltonian reduction picture that we developed permits to understand the exact solvability of the reduced systems, in principle from any point of view ranging from the proof of complete integrability to the explicit construction of the solutions.

We presented new integrable models built on the diagram automorphisms of the real split and compact simple Lie algebras. To illustrate the considerable range of applicability of the dynamical $r$-matrix construction, we now mention further examples that could be developed utilizing $r$-matrices of the form (3.2). For instance, one could apply the construction to the Cartan involutions of the simple real Lie algebras. Cartan involutions are singled out since their use guarantees the definite character of the kinetic energy of the corresponding models. New models are expected to arise from those Cartan involutions that are outer automorphisms of non-split real Lie algebras. In fact, this holds for the real forms $s u^{*}(2 n)$ and $s o(2 p+1,2 q+1)$ 
$(p \neq q)$ of the classical complex Lie algebras and for some real forms of $E_{6}$. A rich set of other examples can be derived by considering direct sum Lie algebras composed of $N>1$ identical copies of a self-dual Lie algebra $\mathcal{A}$. The scalar product of $\mathcal{A}$ induces a scalar product on

$$
\mathcal{G}:=\mathcal{A} \oplus \mathcal{A} \oplus \cdots \oplus \mathcal{A}
$$

in such a way that one obtains a scalar product preserving automorphism $\theta$ of $\mathcal{G}$ by the formula

$$
\theta: \mathcal{G} \rightarrow \mathcal{G}, \quad \theta\left(u_{1}, u_{2}, \ldots, u_{N}\right):=\left(\tau u_{N}, \tau u_{1}, \ldots, \tau u_{N-1}\right),
$$

where $\tau$ is a scalar product preserving automorphism of $\mathcal{A}$. In the simplest case $\tau=\mathrm{id}$ and $\theta$ is the cyclic permutation automorphism. We have inspected this case by taking an arbitrary simple Lie algebra for $\mathcal{A}$ and taking $\mathcal{K}$ as the diagonal embedding of a Cartan subalgebra of $\mathcal{A}$ into $\mathcal{G}$. Then it turned out that the dynamical $r$-matrix construction reproduces (for $\mathcal{A}=A_{n}$ ) certain generalized spin Calogero models found earlier in [32, 33] by different methods. These examples and their generalizations for affine Lie algebras will be further studied elsewhere.

A very interesting question that remains to be investigated is whether all spin Calogero type models that may be associated with arbitrary solutions of CDYBE (2.5) and its spectral parameter dependent version can be understood as Hamiltonian reductions of suitable 'obviously integrable' systems. The quantization of the spin Calogero models is another important problem that appears to be largely open.

Finally, let us discuss the generalization of the construction of Section 2 to dynamical $r$-matrices based on non-Abelian subalgebras, which were introduced in [21] and appeared afterwards in various contexts (see e.g. [42, 43] and references therein). In fact, the main examples of such $r$-matrices are the 'extended versions' of the Alekseev-Meinrenken $r$-matrices (3.2) given on the full fixpoint set, say $\mathcal{G}_{0} \subset \mathcal{G}$, of the automorphism $\theta$ by the formula 27]

$$
R_{\text {ext }}^{\theta}: \check{\mathcal{G}}_{0} \rightarrow \operatorname{End}(\mathcal{G}), \quad q \mapsto R_{\text {ext }}^{\theta}(q):= \begin{cases}f\left(\operatorname{ad}_{q}\right) & \text { on } \mathcal{G}_{0} \\ \frac{1}{2}\left(\left.\theta e^{\operatorname{ad}_{q}}\right|_{\mathcal{G}_{0}^{\perp}}+1\right)\left(\left.\theta e^{\operatorname{ad}_{q}}\right|_{\mathcal{G}_{0}^{\perp}}-1\right)^{-1} & \text { on } \mathcal{G}_{0}^{\perp}\end{cases}
$$

with the analytic function $f(z)=\frac{1}{2} \operatorname{coth} \frac{z}{2}-\frac{1}{z}$. Now, even if $\mathcal{G}_{0}$ is non-Abelian, one can still use (2.9) to define a quasi-Lax operator $L(q, p, \xi)$ on $T^{*} \check{\mathcal{G}}_{0} \times \mathcal{G}^{*} \simeq \check{\mathcal{G}}_{0} \times \mathcal{G}_{0} \times \mathcal{G}$, but then the anomalous term in (2.10) becomes $\nabla_{\chi} R_{\text {ext }}^{\theta}$ with $\chi(q, p, \xi)=[q, p]+\xi_{\mathcal{G}_{0}}$. This statement can be found in [23] and is also readily verified similarly to Proposition 1 . Therefore, in the nonAbelian case one has to impose the constraint $\chi=0$ to get rid of the anomalous term. If a non-Abelian $\mathcal{G}_{0}$ contains an appropriate Abelian $\mathcal{K}$, then $\chi=0$ represents a larger set of constraints than $\xi_{\mathcal{K}}=0$ does, and $\chi=0$ also generates a larger gauge group. In the end, further supposing for simplicity that $\mathcal{G}$ is compact, it is possible to show that the reduced phase space of the 'non-Abelian construction' defined by imposing the first class constraint $\chi=0$ on $T^{*} \check{\mathcal{G}}_{0} \times \mathcal{G}^{*}$ is the same as the one obtained from the corresponding 'Abelian construction' that operates by imposing $\xi_{\mathcal{K}}=0$ on $T^{*} \check{\mathcal{K}} \times \mathcal{G}^{*}$. (If $\mathcal{G}$ is reductive, then $\mathcal{K}$ must be a Cartan subalgebra of $\mathcal{G}_{0}$ for $R^{\theta}(3.2)$ to be well-defined. A slight complication for a non-compact real reductive $\mathcal{G}$ arises since $\mathcal{G}_{0}$ admits non-conjugate Cartan subalgebras in general.) By the 
argument just sketched, we do not expect the $r$-matrices based on non-Abelian subalgebras to lead to interesting new integrable models. This observation motivated us in the first place to restrict ourselves throughout this paper to dynamical $r$-matrices based on Abelian subalgebras. Our argument is closely related to the mapping between different $r$-matrices defined by means of Dirac reduction of the space of dynamical variables as studied in 44]. The connection between the Abelian and non-Abelian variants of the dynamical $r$-matrix construction of spin Calogero type models is elaborated in more detail in [45]

\section{A On the non-degenerate Abelian dynamical $r$-matrices}

In this appendix we show that the $r$-matrices (3.2) found in [27] can be uniquely characterized among the solutions of the CDYBE (with coupling $\nu=1$ ) by requiring the additional properties (A.7) and (A.8) that ensure the equivalence of the Lax equation (2.17) with the Hamilton equation (2.19) upon imposing the constraint $\xi_{\mathcal{K}}=0$ and setting $p=\dot{q}$. The constrained Hamilton equation always implies the Lax equation, but is not necessarily equivalent to it in general. For compact Lie algebras the non-degeneracy condition (A.7) holds automatically, which allows us to obtain a rather strong result about the solutions of the CDYBE in this case.

Before presenting our results we introduce a convenient notion of Cayley transformation. Let $R \in \operatorname{End}(\mathcal{G})$ be an antisymmetric linear operator on a self-dual Lie algebra $\mathcal{G}$ such that the operators $R_{ \pm}:=R \pm \frac{1}{2} \mathrm{id}_{\mathcal{G}}$ are invertible. (This hold for all antisymmetric operators if $\mathcal{G}$ is a real Lie algebra with 'scalar product' $\langle$,$\rangle of definite signature.) We call$

$$
C:=R_{+} R_{-}^{-1}
$$

the Cayley transform of $R$. We then also have the inverse Cayley transformation formula

$$
R=\frac{1}{2}(C+1)(C-1)^{-1}
$$

Note that $C \in \operatorname{End}(\mathcal{G})$ is an 'orthogonal' operator, i.e.,

$$
\langle C X, C Y\rangle=\langle X, Y\rangle, \quad \forall X, Y \in \mathcal{G}
$$

In fact, (A.1) and (A.2) represent a bijective correspondence between the 'antisymmetric' operators $R$ for which $R_{ \pm}$are invertible and the 'orthogonal' operators $C$ that do not admit the eigenvalue +1 . (Up to some signs, this becomes the usual Cayley transformation for $\mathcal{G}=s o(3)$.)

Let $\mathcal{K} \subset \mathcal{G}$ be an arbitrary (not necessarily Abelian) self-dual subalgebra with a non-empty open, connected domain $\check{\mathcal{K}} \subset \mathcal{K}$. Assuming that

$$
R: \check{\mathcal{K}} \rightarrow \operatorname{End}(\mathcal{G}), \quad q \mapsto R(q)
$$

is antisymmetric and $R_{ \pm}(q)$ are invertible for all $q \in \check{\mathcal{K}}$, we introduce the Cayley transform $C: \check{\mathcal{K}} \rightarrow \operatorname{End}(\mathcal{G})$ by pointwise application of (A.1). One can then verify 
Lemma A.1. The map $R$ is $\mathcal{K}$-equivariant, iff its Cayley transform $C$ is $\mathcal{K}$-equivariant, i.e.,

$$
\left(\nabla_{[T, q]} R\right)(q)=\left[\operatorname{ad}_{T}, R(q)\right] \quad \Leftrightarrow \quad\left(\nabla_{[T, q]} C\right)(q)=\left[\operatorname{ad}_{T}, C(q)\right] \quad(\forall T \in \mathcal{K}, \forall q \in \check{\mathcal{K}}) .
$$

$R$ solves the $C D Y B E$ (2.5) with $\nu=1$, iff $C$ satisfies the equation

$$
\begin{aligned}
C(q)[X, Y]- & {[C(q) X, C(q) Y]+(C(q)-1)\langle C(q) X,(\nabla C)(q) Y\rangle } \\
& -\left(\nabla_{(C(q) Y-Y)_{\mathcal{K}}} C\right)(q) X+\left(\nabla_{(C(q) X-X)_{\mathcal{K}}} C\right)(q) Y=0
\end{aligned}
$$

for all $q \in \check{\mathcal{K}}$ and $X, Y \in \mathcal{G}$.

Here, we are interested in antisymmetric $r$-matrices, $R: \check{\mathcal{K}} \rightarrow \operatorname{End}(\mathcal{G})$, defined on an Abelian self-dual subalgebra $\mathcal{K} \subset \mathcal{G}$ that are compatible with the decomposition $\mathcal{G}=\mathcal{K}+\mathcal{K}^{\perp}$ and satisfy the following non-degeneracy condition:

$$
\left.R_{-}(q)\right|_{\mathcal{K}^{\perp}} \text { is invertible } \forall q \in \check{\mathcal{K}} \text {. }
$$

The compatibility really means that $R(q) \mathcal{K} \subset \mathcal{K}$ since then $R(q) \mathcal{K}^{\perp} \subset \mathcal{K}^{\perp}$ follows from the antisymmetry. For simplicity, we assume that the mapping $R: \check{\mathcal{K}} \rightarrow \operatorname{End}(\mathcal{G})$ is smooth or holomorphic for real or complex $\mathcal{G}$, respectively. Without further loss of generality, we can impose the auxiliary condition

$$
\left.R(q)\right|_{\mathcal{K}}=0, \quad \forall q \in \check{\mathcal{K}} .
$$

This can be achieved, because (as is well known and easy to check) the restriction of the operator $R(q)$ to $\mathcal{K}$ is required by the CDYBE to yield an arbitrary closed 2 -form, $F$, on $\check{\mathcal{K}}$ by

$$
F(q)=\sum_{i j}\left\langle T_{i}, R(q) T_{j}\right\rangle d q^{i} \wedge d q^{j}
$$

with a basis $T_{i}$ of $\mathcal{K}$ and corresponding coordinates $q^{i}$ of $q \in \check{\mathcal{K}}$. If (A.7) and (A.8) hold, then we can use the Cayley transform (A.1) to prove the following result.

Proposition A.2. The (smooth or holomorphic) $\mathcal{K}$-equivariant solutions of the CDYBE (2.5) with $\nu=1$, on an Abelian self-dual subalgebra $\mathcal{K} \subset \mathcal{G}$, that are non-degenerate in the sense A.7) and satisfy also (A.8) are precisely the maps of the form

$$
R(q) X=\frac{1}{2}\left(\theta e^{\operatorname{ad}_{q}}+1\right)\left(\left.\left(\theta e^{\operatorname{ad}_{q}}-1\right)\right|_{\mathcal{K}^{\perp}}\right)^{-1} X, \quad \forall X \in \mathcal{K}^{\perp}, q \in \check{\mathcal{K}},
$$

where $\theta$ is a scalar product preserving automorphism of $\mathcal{G}$ for which $\theta X=X$ for any $X \in \mathcal{K}$ and the inverse in (A.10) exists.

Proof. By the Cayley transformation formula (A.1) the assumption $\left.R(q)\right|_{\mathcal{K}}=0$ translates into the equivalent equation $\left.C(q)\right|_{\mathcal{K}}=-\mathrm{id}_{\mathcal{K}}$, and our aim is to determine $\left.C(q)\right|_{\mathcal{K}^{\perp}}$. To do that, first notice that in our case the $\mathcal{K}$-equivariance property (A.5) reads

$$
\left[\operatorname{ad}_{T}, C(q)\right]=0, \quad \forall q \in \check{\mathcal{K}}, \forall T \in \mathcal{K} .
$$


Now, let us turn our attention to the equation (A.6) for $C$. When $X, Y \in \mathcal{K}$, then (A.6) becomes an obvious identity and gives no information. Next, consider the case when $X \in \mathcal{K}$ and $Y \in \mathcal{K}^{\perp}$. Then we easily get

$$
\left(\nabla_{X} C\right)(q) Y=[X, C(q) Y]
$$

We get the same equation when $X \in \mathcal{K}^{\perp}$ and $Y \in \mathcal{K}$, up to change of letters. The most interesting case occurs when $X, Y \in \mathcal{K}^{\perp}$. Then, using the above two relations one finds that (A.6) can be written as

$$
C[X, Y]-[C X, C Y]+2[C X, C Y]_{\mathcal{K}}=0
$$

which is in fact equivalent to the following system of equations

$$
[C X, C Y]_{\mathcal{K}}=[X, Y]_{\mathcal{K}}, \quad[C X, C Y]_{\perp}=C[X, Y]_{\perp}
$$

Now, let us introduce the function

$$
\theta_{\perp}: \check{\mathcal{K}} \rightarrow \operatorname{GL}\left(\mathcal{K}^{\perp}\right), \quad q \mapsto \theta_{\perp}(q):=\left.\left(C(q) e^{-\mathrm{ad}_{q}}\right)\right|_{\mathcal{K}^{\perp}}
$$

Equation (A.12) with (A.11) implies that this function satisfies the differential equation

$$
\left(\nabla_{X} \theta_{\perp}\right)(q)=0, \quad \forall q \in \check{\mathcal{K}}, \forall X \in \mathcal{K} .
$$

Hence, $\theta_{\perp}(q)$ is necessarily constant, $\theta_{\perp}(q)=\theta_{\perp}(\forall q \in \check{\mathcal{K}})$. We extend this constant operator onto the whole Lie algebra $\mathcal{G}$ by the formula

$$
\theta: \mathcal{G} \rightarrow \mathcal{G}, \quad \theta:= \begin{cases}\operatorname{id}_{\mathcal{K}} & \text { on } \mathcal{K}, \\ \theta_{\perp} & \text { on } \mathcal{K}^{\perp}\end{cases}
$$

Then, one readily checks that $C(q)$ satisfies equations (A.3), (A.11), (A.12) and (A.14) if and only if the extended operator $\theta$ (A.17) is a scalar product preserving automorphism of the selfdual Lie algebra $\mathcal{G}$. We have just demonstrated that under our conditions the Cayley transform (A.1) of $R(q)$ has the form

$$
C(q)= \begin{cases}-\mathrm{id}_{\mathcal{K}} & \text { on } \mathcal{K}, \\ \theta e^{\operatorname{ad}_{q}} & \text { on } \mathcal{K}^{\perp},\end{cases}
$$

where $\theta \in \operatorname{Aut}(\mathcal{G})$ is a scalar product preserving automorphism with the property $\left.\theta\right|_{\mathcal{K}}=\operatorname{id}_{\mathcal{K}}$. So, by applying the inverse Cayley transformation (A.2) we obtain the Proposition. Q.E.D.

Now we wish to point out some consequences. First note that if the self-dual, Abelian subalgebra $\mathcal{K} \subset \mathcal{G}$ is maximal as subalgebra, then $R(q) \mathcal{K} \subset \mathcal{K}$ follows from the equivariance property. Thus in this case the $\mathcal{K}^{\perp}$-component of any non-degenerate solution of the CDYBE (with coupling $\nu=1$ ) has the form (A.10), while its $\mathcal{K}$-component is furnished by a closed 2-form $F$ according (A.9). This gives a characterization of the non-degenerate dynamical $r$ matrices on the Cartan subalgebras of the (real or complex) simple Lie algebras. If $\mathcal{K}$ is a Cartan subalgebra of a complex simple $\mathcal{G}$, then all automorphisms that fix $\mathcal{K}$ pointwise have 
the form $\theta=e^{\operatorname{ad}_{v}}$ for a constant $v \in \mathcal{K}$, and Proposition A.2 reproduces a result of Etingof and Varchenko (see Theorem 3.1 in [21]). More interestingly, notice that Proposition A.2 can be applied to the classification of the $r$-matrices on Abelian subalgebras of compact Lie algebras, since in this case $R_{ \pm}$are automatically invertible for any antisymmetric $R$. Hence we obtain the following 'dynamical analogue' of the well-known uniqueness result [46] on constant $r$-matrices of compact Lie algebras.

Corollary A.3. The $\mathcal{K}$-equivariant solutions of the CDYBE (2.5) with $\nu=1$ on a Cartan subalgebra $\mathcal{K}$ of a compact simple Lie algebra $\mathcal{G}$ are precisely the maps $q \mapsto R(q)$ for which $\left.R(q)\right|_{\mathcal{K}}$ corresponds to a closed 2-form by (A.9) and

$$
\left.R(q)\right|_{\mathcal{K}^{\perp}}=\frac{1}{2} \operatorname{coth}\left(\left.\frac{1}{2} \operatorname{ad}_{q+v}\right|_{\mathcal{K}^{\perp}}\right)
$$

with an arbitrary constant $v \in \mathcal{K}$. The smoothness of $R$ requires that $\varphi(q+v) \notin 2 \pi \mathrm{i} \mathbb{Z}$ for any root $\varphi$ with respect to (the complexification of) $\mathcal{K}$.

To obtain the corollary we used that the automorphisms of a compact simple $\mathcal{G}$ whose fixpoint sets contain the Cartan subalgebra $\mathcal{K}$ are of the form $e^{\operatorname{ad}_{v}}$ with some $v \in \mathcal{K}$. It can also be checked that from all (non-degenerate or not) solutions of the CDYBE that exist in the corresponding complex case [21] precisely those giving (A.19) survive the restriction to the compact real form.

For clarity, let us finally remind that in the CDYBE (2.5) the constant $\nu$ can be replaced by $\beta^{2} \nu$ if one replaces $R(q)$ by $\beta R(\beta q)$ with a constant $\beta \neq 0$. If $\nu \neq 0$, then for a complex $\mathcal{G}$ the value of $\nu$ can thus be scaled to 1 , while for a real $\mathcal{G}$ the two different possibilities $\nu=1$ and $\nu=\mathrm{i}$ arise in principle. We have chosen $\nu=1$ since this is required for Proposition 1 . (The same choice is needed for applications in the WZNW model [42.) Up to gauge transformations, for a simple $\mathcal{G}$ all non-constant solutions with $\nu \neq 0$ that are known to us belong to $\nu=1$. However, for a compact $\mathcal{G}$ the constant solutions have $\nu=\mathrm{i}$, and to our knowledge it has not been investigated if $\nu=\mathrm{i}$ is excluded for non-constant solutions of the CDYBE or not.

\section{B Folding by an involutive diagram automorphism}

For convenience, we here summarize the 'folding procedure' referred to in Section 5.

We denote by $\tau$ the non-trivial involutive automorphism of the Dynkin diagram of $\mathcal{A} \in$ $\left\{A_{n}, D_{n}, E_{6}\right\}$ as well as its extension to $\mathcal{A}$ and to the root system $\Phi$. Let us decompose the set of positive roots in the form

$$
\Phi_{+}=\Xi \cup \Psi \cup \tau(\Psi)
$$

where the elements of $\Xi$ are fixed by $\tau$, and the 2-point orbits of $\tau$ in $\Phi_{+}$are represented by $\Psi$. Since $\tau$ is a Cartan preserving involution of $\mathcal{A}$, any choice of root vectors enjoys the property $\tau\left(X_{\varphi}\right)=c_{\varphi} X_{\tau(\varphi)}$ with some constants $c_{\varphi}$ subject to $c_{\varphi} c_{\tau(\varphi)}=1$. If the root vectors are chosen as described in Section 5 , then these constants satisfy also the relations $c_{-\varphi}=c_{\varphi}$ and $c_{\varphi} c_{-\varphi}=1$. The first relation follows since $\tau$ commutes with the Chevalley automorphism $\sigma$, and the second 
one is implied by $1=\left\langle X_{\varphi}, X_{-\varphi}\right\rangle=\left\langle\tau\left(X_{\varphi}\right), \tau\left(X_{-\varphi}\right)\right\rangle$. As a consequence, $c_{\varphi}^{2}=1$ and $c_{\varphi}=c_{\tau(\varphi)}$ for any $\varphi \in \Phi$. We can decompose $\Xi$ as $\Xi=\Xi^{+} \cup \Xi^{-}$with

$$
\Xi^{ \pm}:=\left\{\varphi \in \Phi_{+} \mid \tau(\varphi)=\varphi, c_{\varphi}= \pm 1\right\}
$$

For any $\varphi \in \Phi$ denote by $\bar{\varphi}$ the restriction of $\varphi$ to $\mathcal{H}^{+}$. It is known (and is easy to check) that $\bar{\varphi} \neq 0 \forall \varphi \in \Phi$ and different $\tau$-orbits in $\Phi$ yield different functionals on $\mathcal{H}^{+}$upon restriction.

In fact, a system of positive roots of $\left(\mathcal{H}^{+}, \mathcal{A}^{+}\right)$, introduced in Section 5 , is provided by

$$
\Delta_{+}=\left\{\bar{\varphi} \mid \varphi \in \Psi \cup \Xi^{+}\right\},
$$

and the positive weights of $\left(\mathcal{H}^{+}, \mathcal{A}^{-}\right)$are furnished by

$$
\Gamma_{+}=\left\{\bar{\varphi} \mid \varphi \in \Psi \cup \Xi^{-}\right\} .
$$

The root vectors used in Section 5 can be taken to be

$$
X_{ \pm \bar{\varphi}}^{+}=\frac{1}{\sqrt{2}}\left(X_{ \pm \varphi}+\tau\left(X_{ \pm \varphi}\right)\right) \quad \forall \varphi \in \Psi, \quad X_{ \pm \bar{\varphi}}^{+}=X_{ \pm \varphi} \quad \forall \varphi \in \Xi^{+}
$$

and the weight vectors can be chosen as

$$
X_{ \pm \bar{\varphi}}^{-}=\frac{1}{\sqrt{2}}\left(X_{ \pm \varphi}-\tau\left(X_{ \pm \varphi}\right)\right) \quad \forall \varphi \in \Psi, \quad X_{ \pm \bar{\varphi}}^{-}=X_{ \pm \varphi} \quad \forall \varphi \in \Xi^{-} .
$$

It is easy to see that the generators provided by this 'folding procedure' have the properties claimed in Section 5.

In the most complicated example $\mathcal{A}=A_{2 n}$, we have $\Phi_{+}=\left\{\epsilon_{k}-\epsilon_{l} \mid 1 \leq k<l \leq(2 n+1)\right\}$. With the usual conventions $\tau: \epsilon_{k} \mapsto-\epsilon_{2 n+2-k}(\forall k=1, \ldots, 2 n+1)$. Then we obtain

$$
\begin{gathered}
\Psi=\left\{\epsilon_{k}-\epsilon_{l}, \epsilon_{k}-\epsilon_{2 n+2-l}, \epsilon_{m}-\epsilon_{n+1} \mid 1 \leq k<l \leq n, 1 \leq m \leq n\right\}, \\
\Xi=\Xi^{-}=\left\{\epsilon_{m}-\epsilon_{2 n+2-m} \mid 1 \leq m \leq n\right\} .
\end{gathered}
$$

The restriction of the above elements of $\Psi$ and $\Xi^{-}$to $\mathcal{H}^{+}$gives

$$
\bar{\epsilon}_{k}-\bar{\epsilon}_{l}=e_{k}-e_{l}, \quad \bar{\epsilon}_{k}-\bar{\epsilon}_{2 n+2-l}=e_{k}+e_{l}, \quad \bar{\epsilon}_{m}-\bar{\epsilon}_{n+1}=e_{m}, \quad \bar{\epsilon}_{m}-\bar{\epsilon}_{2 n+2-m}=2 e_{m} .
$$

In the usual realization $A_{2 n}=s l_{2 n+1}, \tau: E_{a, b} \mapsto(-1)^{b-a+1} E_{2 n+2-b, 2 n+2-a}$ with the elementary matrices $E_{a, b}$ for $a, b=1, \ldots, 2 n+1$. Then one readily verifies (B.9) by using that $\epsilon_{a}$ maps $Q=\operatorname{diag}\left(Q_{1}, \ldots, Q_{2 n+1}\right) \in \mathcal{H}$ to $Q_{a}$ for $a=1, \ldots, 2 n+1$ and $e_{k}$ maps $q=\operatorname{diag}\left(q_{1}, \ldots, q_{n}, 0,-q_{n}, \ldots,-q_{1}\right) \in \mathcal{H}^{+}$to $q_{k}$ for $k=1, \ldots, n$. The claimed properties of the root and weight vectors can also be checked on this model, but, of course, all the calculations can be done in a model independent manner as well.

Acknowledgements. The work of L.F. was supported in part by the Hungarian Scientific Research Fund (OTKA) under the grants T043159, T049495, M045596 and by the EU networks 'EUCLID' (contract number HPRN-CT-2002-00325) and 'ENIGMA' (contract number MRTNCT-2004-5652). B.G.P. is grateful for support by a CRM-Concordia Postdoctoral Fellowship and he especially wishes to thank J. Harnad for hospitality in Montreal. We are also indebted to J. Balog and I. Marshall for useful comments on the manuscript. 


\section{References}

[1] F. Calogero, Solution of the one-dimensional $N$-body problem with quadratic and/or inversely quadratic pair potentials, J. Math. Phys. 12 (1971) 419-436.

[2] B. Sutherland, Exact results for a quantum many body problem in one dimension, Phys. Rev. A 4 (1971) 2019-2021.

[3] J. Moser, Three integrable Hamiltonian systems connected with isospectral deformations, Adv. Math. 16 (1975) 197-220.

[4] A.M. Perelomov, Integrable Systems of Classical Mechanics and Lie Algebras, Birkhäuser, 1990.

[5] N. Nekrasov, Infinite-dimensional algebras, many-body systems and gauge theories, pp. 263299 in: Moscow Seminar in Mathematical Physics, AMS Transl. Ser. 2, A.Yu. Morozov and M.A. Olshanetsky (Editors), Amer. Math. Soc., 1999.

[6] J.F. van Diejen and L. Vinet (Editors), Calogero-Moser-Sutherland Models, Spinger, 2000.

[7] B. Sutherland, Beautiful Models, World Scientific, 2004.

[8] M.A. Olshanetsky and A.M. Perelomov, Completely integrable Hamiltonian systems connected with semisimple Lie algebras, Invent. Math. 37 (1976) 93-108.

[9] J. Gibbons and T. Hermsen, A generalisation of the Calogero-Moser system, Physica D 11 (1984) 337-348.

[10] S.N.M. Ruijsenaars and H. Schneider, A new class of integrable models and their relation to solitons, Ann. Phys. (NY) 170 (1986) 370-405.

[11] M.A. Olshanetsky and A.M. Perelomov, Explicit solution of the Calogero model in the classical case and geodesic flows on symmetric spaces of zero curvature, Lett. Nouvo Cim. 16 (1976) 333-339; Explicit solutions of some completely integrable systems, Lett. Nouvo Cim. 17 (1976) 97-101.

[12] D. Kazhdan, B. Kostant and S. Sternberg, Hamiltonian group actions and dynamical systems of Calogero type, Comm. Pure Appl. Math. XXXI (1978) 481-507.

[13] O. Babelon and C.-M. Viallet, Hamiltonian structures and Lax equations, Phys. Lett. B 237 (1990) 411-416.

[14] J. Avan and M. Talon, Classical R-matrix structure for the Calogero model, Phys. Lett. B 303 (1993) 33-37, hep-th/9210128.

[15] E.K. Sklyanin, Dynamical r-matrices for the elliptic Calogero-Moser model, Alg. and Anal. 6 (1994) 227-237, hep-th/9308060. 
[16] H.W. Braden and T. Suzuki, R-matrices for elliptic Calogero-Moser models, Lett. Math. Phys. 30 (1994) 147-158, hep-th/9309033.

[17] E. Billey, J. Avan and O. Babelon, The r-matrix structure of the Euler-Calogero-Moser model, Phys. Lett. A 186 (1994) 114-118, hep-th/9312042; Exact Yangian symmetry in the classical Euler-Calogero-Moser model, Phys. Lett. A 188 (1994) 263-271, hep-th/9401117.

[18] J. Avan, O. Babelon and E. Billey, The Gervais-Neveu-Felder equation and the quantum Calogero-Moser systems, Commun. Math. Phys. 178 (1996) 281-299, hep-th/9505091.

[19] J. Balog, L. Dabrowski and L. Fehér, Classical r-matrix and exchange algebra in WZNW and Toda theories, Phys. Lett. B 244 (1990) 227-234.

[20] G. Felder, Conformal field theory and integrable systems associated to elliptic curves, pp. 1247-1255 in: Proc. ICM Zürich, 1994 (Birkhäuser, 1994), hep-th/9407154.

[21] P. Etingof and A. Varchenko, Geometry and classification of solutions of the classical dynamical Yang-Baxter equation, Commun. Math. Phys. 192 (1998) 77-120, q-alg/9703040.

[22] L.-C. Li and P. Xu, Spin Calogero-Moser systems associated with simple Lie algebras, C. R. Acad. Sci. Paris Serie I, Vol 331, No 1 (2000) 55-61, math.SG/0009180, Integrable spin Calogero-Moser systems, Commun. Math. Phys. 231 (2002) 257-286, math.QA/0105162.

[23] L.-C. Li, A family of hyperbolic spin Calogero-Moser systems and the spin Toda lattices, Comm. Pure Appl. Math. LVII (2004) 791-832, math-ph/0506028.

[24] L.-C. Li, Coboundary dynamical Poisson groupoids and integrable systems, Int. Math. Res. Not. 2003, no. 51, 2725-2746, math-ph/0506027.

[25] L.-C. Li, Poisson involutions, spin Calogero-Moser systems associated with symmetric Lie subalgebras and the symmetric space spin Ruijsenaars-Schneider model, math-ph/0506025.

[26] L.-C. Li, A class of integrable spin Calogero-Moser systems II: exact solvability, math-ph/0506026.

[27] A. Alekseev and E. Meinrenken, Clifford algebras and the classical dynamical Yang-Baxter equation, Math. Res. Lett. 10 (2003) 253-268, math.RT/0209347.

[28] N. Reshetikhin, Degenerate integrability of spin Calogero-Moser systems and the duality with the spin Ruijsenaars systems, Lett. Math. Phys. 63 (2003) 55-71, math.QA/0202245.

[29] D. Alekseevsky, A. Kriegl, M. Losik and P.W. Michor, The Riemannian geometry of orbit spaces. The metric, geodesics, and integrable systems, Publ. Math. Debrecen 62 (2003) 247-276, math.DG/0102159.

[30] J. Arnlind and J. Hoppe, Eigenvalue-dynamics off the Calogero-Moser system, Lett. Math. Phys. 68 (2004) 121-129. 
[31] S. Hochgerner, Singular cotangent bundle reduction and spin Calogero-Moser systems, math.SG/0411068.

[32] J. Blom and E. Langmann, Novel integrable spin-particle models from gauge theories on a cylinder, Phys. Lett. B 429 (1998) 336-342, solv-int/9804007; Finding and solving CalogeroMoser type systems using Yang-Mills gauge theories, Nucl. Phys. B 563 (1999) 506-532, math-ph/9909019

[33] A.P. Polychronakos, Generalized Calogero-Sutherland systems from many-matrix models, Nucl. Phys. B 546 (1999) 495-502, hep-th/9806189.

[34] P. Etingof and O. Schiffmann, On the moduli space of classical dynamical r-matrices, Math. Res. Lett. 8 (2001) 157-170, math.QA/0005282.

[35] R. Sjamaar and E. Lerman, Stratified symplectic spaces and reduction, Ann. Math. 134 (1991) 375-422.

[36] J.-P. Ortega and T.S. Ratiu, Momentum Maps and Hamiltonian Reduction, Birkhäuser, 2004.

[37] P. Libermann and C.-M. Marle, Symplectic Geometry and Analytical Mechanics, D. Reidel Publ. Company, 1986.

[38] S. Mohrdieck and R. Wendt, Integral conjugacy classes of compact Lie groups, Manuscripta Math. 114 (2004) 531-547, math.QA/0303118

[39] V.G. Kac, Infinite Dimensional Lie Algebras, third edition, Cambride, 1990.

[40] G. Warner, Harmonic Analysis on Semi-Simple Lie Groups I, Springer, 1972.

[41] V.S. Gerdjikov, A. Kyuldjiev, G. Marmo and G. Vilasi, Real Hamiltonian forms of Hamiltonian systems, nlin.SI/0310005.

[42] L. Fehér, Dynamical r-matrices and Poisson-Lie symmetries in the chiral WZNW model, Review presented at the Workshop on Integrable Theories, Solitons and Duality, IFT Sao Paulo, July 2002, JHEP Proceedings, PoS(unesp2002)012, hep-th/0212006.

[43] B. Enriquez and P. Etingof, Quantization of classical dynamical r-matrices with nonAbelian base, Commun. Math. Phys. 254 (2005) 603-650, math.QA/0311224.

[44] L. Fehér, A. Gábor and B.G. Pusztai, On dynamical r-matrices obtained from Dirac reduction and their generalizations to affine Lie algebras, J. Phys. A 34 (2001) 7235-7248, math-ph/0105047

[45] L. Fehér and B.G. Pusztai, Spin Calogero models and dynamical r-matrices, to appear in proceedings of Symposium QTS-4, Varna (Bulgaria), August 2005, math-ph/0511059.

[46] Ya. S. Soibelman, Irreducible representations of the function algebra on the quantum group $S U(n)$, and Schubert cells, Soviet Math. Dokl. 40 (No. 1) (1990) 34-38. 